\title{
A CLASSIFICATION THEOREM FOR SKT-MODULES
}

\author{
BRIAN D. WICK
}

\begin{abstract}
In this paper a class of Abelian groups which includes the torsion totally projective groups, $S$-groups, and balanced projectives is studied. It is shown that this class of groups has a complete set of invariants.
\end{abstract}

If $p$ is a prime number then the ring of all rational numbers $a / b$ with $b$ relatively prime to $p$ will be denoted by $Z_{p}$.

The category of $Z_{p}$-modules are those Abelian groups with the property that multiplication by a prime other than the prime $p$ is an automorphism of the group. If $M$ is a $Z_{p}$-module then (i) the torsion submodule of $M$ is the maximal torsion subgroup of $M$; (ii) $M$ is torsion-free if and only if the torsion submodule of $M$ is (0); (iii) $M$ is reduced if and only if $M$ is a reduced group; and (iv) $M$ is divisible if and only if $M$ is a divisible group.

$Z$ and $Q$ will denote the groups of integers and rational numbers respectively. The group $\operatorname{Ext}\left(Q / Z_{p}, *\right)$ will be denoted by $c(*)$.

The limit ordinal $\lambda$ is a limited ordinal cofinal with $\omega$ if there is a sequence of smaller ordinals $\beta_{i}$ such that $\lambda=\sup \beta_{i}$. Otherwise $\lambda$ is said to be not cofinal with $\omega$.

Three families of invariants $u, h$, and $k$ are defined for the group $G$ as follows. The invariant $u\left(p^{\alpha}, G\right)$ (the Ulm invariant) for the prime $p$ and ordinal $\alpha$ is the dimension of the $Z / p Z$-vector space $\left(p^{\alpha} G\right)[p] /\left(p^{\alpha+1} G\right)[p] \equiv U\left(p^{\alpha}, G\right)$. The invariant $h\left(p^{\beta}, G\right)$ for the prime $p$ and ordinal $\beta$ is the dimension of the $Z / p Z$-vector space $p^{\beta} G /\left(p^{\beta+1} G+T\right)$, where $T$ is the maximal torsion subgroup of $p^{\beta} G$. The invariant $k\left(p^{\lambda}, G\right)$ for the prime $p$ and limit ordinal $\lambda$ such that $\lambda$ is not cofinal with $\omega$, is the dimension of the $Z / p Z$-vector space $K\left(p^{\lambda}, G\right) \equiv$ $p^{\lambda} c\left(G / p^{\lambda} G\right) / p^{\lambda+1} c\left(G / p^{\lambda} G\right)$.

Warfield [2] and [3] showed that the family of invariants $u$ and $h$ classify the balanced projectives, and the family of invariants $u$ and $k$ classify the $S$-groups. Noting that the family of invariants $h$ are 0 on the $S$-groups, and the family of invariants $k$ are 0 on the balanced projectives, it was conjectured that the family of invariants $u, h$ and $k$ could be used to classify those groups which are the direct sum of $S$-groups and balanced projectives. These groups are called SKT-modules. It will be shown in [4] that SKT-modules are projective relative to a well-defined class of sequences. It then follows that the SKT-modules form a class of groups

Received by the editors April 20, 1979 and, in revised form, October 5, 1979.

AMS (MOS) subject classifications (1970). Primary 20F15; Secondary 20K25, 20E99, 20 K99. 
which contains the $S$-groups and balanced projectives, have a projective characterization, and a complete family of invariants.

For the limit ordinal $\lambda$, a $Z_{p}$-module $M$ is a $\lambda$-elementary balanced projective if and only if $p^{\lambda} M \cong Z_{p}$ and $M / p^{\lambda} M$ is a totally projective $p$-group. The torsion subgroup of $M$ is called a $\lambda$-elementary $S$-group. A $Z_{p}$-module $M$ is a balanced projective if and only if it is isomorphic to the direct sum of a totally projective $p$-group and $\lambda$-elementary balanced projectives for various limit ordinals $\lambda$. The torsion subgroup of a balanced projective is called an $S$-group.

Definition. A module $M$ is an SKT-module if and only if there is a balanced projective $K$ and an $S$-group $S$ such that $M$ is isomorphic to $K \oplus S$.

LEMMA. If $K$ is a balanced projective and $\lambda$ is a limit ordinal not cofinal with $\omega$, then $k\left(p^{\lambda}, K\right)=0$.

Proof. Write

$$
K \cong T \oplus\left(\bigoplus_{\beta \in \Gamma} M_{\beta}\right)
$$

where $T$ is a totally projective $p$-group and $M_{\beta}$ is the direct sum of $\beta$-elementary balanced projectives. Let $\lambda$ be a limit ordinal such that $\lambda$ is not cofinal with $\omega$. If $K \cong A_{\lambda} \oplus B_{\lambda}$ where $A_{\lambda}$ is the direct sum of $T$ and $M_{\beta}$ where $\beta>\lambda$, and $B_{\lambda}$ is the direct sum of all $M_{\beta}$ where $\beta<\lambda$, then $K / p^{\lambda} K \simeq A_{\lambda} / p^{\lambda} A_{\lambda} \oplus B_{\lambda}$. Since $A_{\lambda} / p^{\lambda} A_{\lambda}$ is a totally projective $p$-group, $p^{\lambda} c\left(A_{\lambda} / p^{\lambda} A_{\lambda}\right)=0$ by $[1,3.10]$. If $S$ is the maximal torsion subgroup of $B_{\lambda}$ then $S$ is the direct sum of $p$-groups of length less than $\lambda$ and $0=p^{\lambda} c(S)=p^{\lambda} c\left(B_{\lambda}\right)$ by $[1,3.10]$. It has therefore been shown that $p^{\lambda} c\left(K / p^{\lambda} K\right)=0$ and $k\left(p^{\lambda}, K\right)=0$.

THEOREM. If $A$ and $B$ are SKT-modules such that $u\left(p^{\alpha}, A\right)=u\left(p^{\alpha}, B\right), h\left(p^{\beta}, A\right)$ $=h\left(p^{\beta}, B\right)$, and $k\left(p^{\lambda}, A\right)=k\left(p^{\lambda}, B\right)$ for all ordinals $\alpha, \beta$ and $\lambda$ such that $\beta$ and $\lambda$ are limit ordinals and $\lambda$ is not cofinal with $\omega$, then $A$ is isomorphic to $B$.

Proof. Since $A$ and $B$ are SKT-modules, $A \cong K \oplus S$ and $B \simeq K \oplus \underline{S}$ where $K$ and $K$ are balanced projectives, and $S$ and $S$ are $S$-groups. It can be assumed that $S \cong \bigoplus_{\lambda \in \Lambda} S_{\lambda}$ and $S \cong \bigoplus_{\lambda \in \Delta} S_{\lambda}$ where $S_{\lambda}$ and $\underline{S}_{\lambda}$ are the direct sum of $\lambda$-elementary $S$-groups, and if $\lambda \in \Lambda$ or $\lambda \in \Delta$ then $\lambda$ is not cofinal with $\omega$. Since $k\left(p^{\lambda}, A\right)=$ $k\left(p^{\lambda}, S\right)=k\left(p^{\lambda}, B\right)=k\left(p^{\lambda}, \underline{S}\right), \Lambda=\Delta \equiv \nabla$. For each $\lambda \in \nabla$, let $S_{\lambda}$ and $\underline{S}_{\lambda}$ be the torsion subgroups of the balanced projectives $N_{\lambda}$ and $N_{\lambda}$ respectively. Furthermore, it will be assumed that $N_{\lambda}$ and $N_{\lambda}$ are direct sums of $\lambda$-elementary balanced projectives. Note that for each $\lambda \in \nabla, \bar{p}^{\lambda} N_{\lambda} \cong p^{\lambda} N_{\lambda}$ because $h\left(p^{\lambda}, N_{\lambda}\right)=k\left(p^{\lambda}, S_{\lambda}\right)$ $=k\left(p^{\lambda}, A\right)=k\left(p^{\lambda}, B\right)=k\left(p^{\lambda}, S_{\lambda}\right)=h\left(p^{\lambda}, \bar{N}_{\lambda}\right)$, [3, 2.3]. Let $M \cong K \oplus$ $\left(\bigoplus_{\lambda \in \nabla} N_{\lambda}\right)$ and $M \cong K \oplus\left(\bigoplus_{\lambda \in \nabla} \bar{N}_{\lambda}\right)$. Since $K$ and $K$ are balanced projectives, then $K \cong T \oplus\left(\bar{\oplus}_{\beta \in \Gamma} K_{\beta}\right)$ and $K \cong T \bigoplus\left(\bigoplus_{\beta \in \psi} \underline{K}_{\beta}\right)$, where $T$ and $\underline{T}$ are totally projective $p$-groups, and $K_{\beta}$ and $\bar{K}_{\beta}$ are the direct sum of $\beta$-elementary balanced projectives. Since $h\left(p^{\beta}, K\right)=h\left(\bar{p}^{\beta}, A\right)=h\left(p^{\beta}, B\right)=h\left(p^{\beta}, K\right), \Gamma=\psi \equiv \Omega$, furthermore, if $\beta \in \Omega$ then $p^{\beta} K_{\beta} \cong p^{\beta} \underline{K}_{\beta}$. 
Let

$$
N=\left(\bigoplus_{\beta \in \Omega} p^{\beta} K_{\beta}\right) \oplus\left(\bigoplus_{\lambda \in \nabla} p^{\lambda} N_{\lambda}\right) \text { and } \underline{N}=\left(\bigoplus_{\beta \in \Omega} p^{\beta} \underline{K}_{\beta}\right) \oplus\left(\bigoplus_{\lambda \in \nabla} p^{\lambda} \underline{N} \lambda\right) \text {. }
$$

Then there is a height preserving isomorphism $\phi: N \rightarrow N$ such that $\phi$ takes $p^{\beta} K_{\beta}$ onto $p^{\beta} K_{\beta}$ and $p^{\lambda} N_{\lambda}$ onto $p^{\lambda} \underline{N}_{\lambda}$ for each $\beta \in \Omega$ and $\lambda \in \bar{\nabla}$. Note that $N$ is a $p$-nice submodule of $M$ and $N$ is a $p$-nice submodule of $M$. In addition, $M / N$ and $M / N$ are totally projective $p$-groups. For each $\alpha, U\left(p^{\alpha}, M\right) / I\left(p^{\alpha}, \overline{N)} \cong\right.$ $U\left(p^{\alpha}, M\right) / I\left(p^{\alpha}, N\right)$ since $M$ and $M$ have the same Ulm invariants and $I\left(p^{\alpha}, N\right)=$ $I\left(p^{\alpha}, \bar{N}\right)=0$. The map $\phi$ extends to an isomorphism $\phi^{*}$ of $M$ onto $M[3,1.2]$. The module $A$ can be identified with the submodule consisting of all elements $g$ in $M$ such that for some integer $n, p^{n} g$ is in the submodule $\bigoplus_{\beta \in \Omega} p^{\beta} K_{\beta}$. The module $B$ can be identified with a similar submodule of $M$. Since the isomorphism $\phi^{*}$ takes the submodule $\bigoplus_{\beta \in \Omega} p^{\beta} K_{\beta}$ onto $\bigoplus_{\beta \in \Omega} p^{\beta} K_{\beta}, \bar{\phi}^{*}$ will take $A$ onto $B$.

\section{REFERENCES}

1. R. Nunke, Homology and direct sums of countable Abelian groups, Math. Z. 101, (1967), 182-212.

2. R. B. Warfield, Jr., A classification theorem for Abelian p-groups, Trans. Amer. Math. Soc. 210 (1975), 149-168.

3. __ Classification theory of Abelian groups. I, Balanced projectives. Trans. Amer. Math. Soc. 222 (1976), 33-63.

4. B. D. Wick, A projective characterization for SKT-modules, Proc. Amer. Math. Soc. 80 (1980), $39-43$.

Department of Mathematics, University of Alaska, ANChorage, Alaska 99504 